\title{
Simulated Annealing-Based Advanced Spectrum Management Methodology for WCDMA Systems
}

\author{
J. Nasreddine, J. Pérez-Romero, O. Sallent, R. Agustí \\ Dept. TSC, Universitat Politècnica de Catalunya (UPC) \\ c/ Jordi Girona, 1-3, Campus Nord, Barcelona, Spain \\ [jnassred, jorperez, sallent, ramon]@tsc.upc.edu
}

\begin{abstract}
In this paper, we propose a new Advanced Spectrum Management (ASM) methodology for WCDMA systems using simulated annealing and the concept of coupling matrix, which is able to capture inter-cell interactions. The proposed methodology takes into account the fact that each cell can be associated to more than one carrier. Simulation results show that the proposed methodology increases spectrum efficiency while guaranteeing the requested QoS levels. Moreover, it allows releasing carriers in some cells, so that they could be eventually used by e.g. secondary cognitive radio users exploiting the flexible spectrum allocation and opportunistic spectrum access.
\end{abstract}

Key words: Coupling matrix, simulated annealing, advanced spectrum management, WCDMA

\section{INTRODUCTION}

The last few years have witnessed a fast pace in the development of Advanced Spectrum Management (ASM) methodologies [1]-[8] paving the way to free-for-all spectrum access with opportunistic spectrum usage [1]. These methodologies exploit the new degree of flexibility introduced by the emerging vision of regulatory bodies [9] about spectrum pooling and sharing in composite networks where different radio access technologies (RAT) and different operators coexist [10]-[12]. Herein, an ASM methodology executed at the planning phase could deal with scenarios in which the traffic either remains constant or changes in a predictable way at known periods of time while a dynamic ASM methodology is necessary to cope with space/time traffic unexpected variations. In both cases, ASM methodologies allow a more efficient use of the spectrum by using intelligent spectrum allocation over cells as it was shown in [13]-[15] for WCDMA networks.

The ASM methodology concerns traffic changes of medium time scales (e.g. minutes or hours) that affect a specific area of the network and aims, in a given RAT, at ameliorating spectrum efficiency by finding the best carrier allocation to cells. Moreover, ASM methodologies should be able to detect the limits of the network to support the existing traffic with its allocated carriers. On the one hand, the ASM methodology should be able to ask for more carriers if the required number of carriers is higher than the actual number of carriers associated to the system. On the other hand, it should be able to put some carriers in a secondary market (e.g. for opportunistic radio usage) if the required number of carriers is lower than the actual number of carriers in order to utilize efficiently these scarce and expensive resources.

Therefore, ASM methodologies aim at finding the minimum number of carriers needed by each cell and the best mobile distribution over carriers that satisfies operator policies. These objectives should be reached while the QoS levels of the served users are kept at the requested level.

In this paper, we formulate the problem of finding efficient spectrum allocation in the uplink of a WCDMA system with multiple carriers, as a step forward towards a generic ASM methodology for composite networks. The proposed methodology is based on the so-called coupling matrix [13] that is able to reflect inter-cell interactions using measurable parameters, and uses simulated annealing meta-heuristic to find near-optimum allocations. Simulated annealing [16] is a general technique dedicated to find a good solution to difficult optimization problems by avoiding local optima. The fundamental idea is to allow moves that lead to worse quality with a time-decreasing probability than the current solution in order to escape from local optima.

The proposed methodology could be integrated in the planning tool to find an efficient spectrum allocation corresponding to periodic traffic changes and it could also be used to dynamically change the carrier allocation in the context of cognitive networks using appropriate triggering events (e.g. QoS indicators, etc.) as it was indicated in [14]. Previous works of the authors in [13]-[15] considered the situation in which only one carrier per cell was allocated. In this paper, the previous work is extended to account for the more general situation in which several carriers per cell can be allocated depending on traffic demands. Furthermore, the proposed strategy is developed from the perspective of releasing significant pieces of spectrum in some cells so that they can be used by cognitive radio users.

The remaining of this paper is organized as follows. First, we introduce the coupling matrix in section 2 . The coupling matrix is used in section 3 in order to develop a simulated annealing-based ASM methodology that enables the system to locally saving some of the allocated carriers (i.e. in some geographical zones). Then, some practical considerations are introduced in section 4 and the performance of the proposed methodology is studied in section 5. Finally, we conclude with useful remarks and future works. 


\section{COUPLING MATRIX}

We consider a WCDMA system with $K$ cells and $F_{\text {all }}$ carriers reflecting that operators use to have more than one frequency carrier in current WCDMA systems. The set of all cells is called $\Lambda=\{j: j \in\{1,2, \ldots, K\}\}$ and the set of allocated carriers to the system is called $\Phi=\left\{f: f \in\left\{1,2, \ldots, F_{\text {all }}\right\}\right\}$. Moreover, we call $\Lambda_{f}$ the set of cells using carrier $f$ and $F_{j}$ the number of carriers allocated to cell $j$.

Coupling matrix has been introduced in [13] and it is computed by assessing a relationship between the total received powers in uplink using $E_{\mathrm{b}} / N_{\mathrm{o}}$ definition:

$$
\left(\frac{E_{\mathrm{b}}}{N_{\mathrm{o}}}\right)_{i_{j}}=\frac{P_{j, i_{j}} \Theta_{i_{j}}}{\sum_{k_{j}=1}^{n_{j}} P_{j, k_{j}}-P_{j, i_{j}}+\chi_{j}+N_{T}}
$$

where $i_{j}$ is the $i$-th user of the $j$-th cell, $n_{j}$ is the number of users connected to cell $j, \Theta_{i_{j}}$ is the spreading factor of mobile $i_{j}$ in uplink (i.e. the ratio between chip rate and bit rate), $P_{j, i_{j}}$ is the useful received power by cell $j$ from mobile $i_{j}, \chi_{j}$ represents the inter-cell interference experienced by mobile $i_{j}$ and $N_{T}$ is the background noise power in uplink. Moreover, $I_{j}$ stands for the total power received by cell $j$ and it is given by:

$$
I_{j}=\chi_{j}+\sum_{k_{j}=1}^{n_{j}} P_{j, k_{j}}+N_{T}
$$

It was shown in [13] that, for a given carrier, vector $\boldsymbol{I}=\left(I_{1}, I_{2}, \ldots, I_{K}\right)^{\mathrm{T}}$ of total received powers in uplink satisfying $E_{\mathrm{b}} / N_{\mathrm{o}}$ constraints can be written in the following matrix form:

$$
\boldsymbol{I}=\boldsymbol{C I}+\boldsymbol{P}_{N}
$$

where $\boldsymbol{C}$ and $\boldsymbol{P}_{\boldsymbol{N}}$ are respectively the $K \mathrm{x} K$ coupling matrix and the $K \times 1$ noise vector defined by their elements:

$$
C_{j, l}=\left\{\begin{array}{cc}
0 & \text { if } l=j \\
\frac{S_{l, j}}{1-S_{j, j}} & \text { otherwise }
\end{array} \quad P_{N, j}=\frac{N_{T}}{1-S_{j, j}}\right.
$$

where $S_{l, j}$ is the impact of users of cell $l$ on cell $j$ :

$$
S_{l, j}=\sum_{i_{l}=1}^{n_{l}} \frac{1}{\varepsilon_{i_{l}}} \frac{L_{i_{l}, l}}{L_{i_{l}, j}} .
$$

where $L_{i, j}$ is the total path loss including antenna gains of mobile $i_{l}$ towards cell $j$ and $\varepsilon_{i_{l}}$ is defined by:

$$
\varepsilon_{i_{l}}=1+\frac{\Theta_{i_{l}}}{\left(E_{\mathrm{b}} / N_{\mathrm{o}}\right)_{i_{l}}}
$$

Each element of matrix $\boldsymbol{C}$ represents the variation of the total power received in uplink by one cell as a response to the variation in the total power received by another cell [13]. It is easy to show that matrix $\boldsymbol{C}$ is a non-negative irreducible matrix using the same methodology of [17]. Therefore, matrix $\boldsymbol{C}$ has interesting properties that can be used in the ASM methodology. In [13], we have shown that a non-constrained system (i.e. a system without power limitation) is able to serve all users with the required $E_{\mathrm{b}} / N_{\mathrm{o}}$ using finite positive powers if and only if the spectral radius $\rho(\boldsymbol{C})$ of the corresponding coupling matrix $\boldsymbol{C}$ (i.e. the eigenvalue with the maximum modulus) is strictly less than unity. When power constraints are added, the value of the spectral radius should be much lower than unity. Therefore, the spectral radius could be considered as a first constraint to system feasibility since it is highly related to interference and transmitted power patterns , and thus to system performance.

In a system with several carriers, a coupling matrix is associated to each carrier. The associated matrix to carrier $f$ is called $\boldsymbol{C}^{(f)}$ and only includes the elements corresponding to cells of set $\Lambda_{f}$ that are associated to carrier $f$. Therefore, the different carriers will have generally coupling matrices with different sizes. In order to map the cell indices of the total coupling matrix $\boldsymbol{C}$ to the indices of matrix elements in the different carriers, we define the set of mapping relations $\mathcal{M}_{f}$ :

$$
\mathcal{M}_{f}(j)=j-K_{f, j}
$$

where $K_{f, j}$ is the number of cells that are not associated to carrier $f$ and have a smaller index than $j$ :

$$
K_{f, j}=\left|\left\{l \in \Lambda-\Lambda_{f}: l<j\right\}\right|
$$

where $|$.$| is the cardinality of a set. Thus, the corresponding$ element reflecting the interaction between cell $j$ and $l$ in $C^{(f)}$ is $C_{\mathcal{M}_{f}(j), \mathcal{M}_{f}(l)}^{(f)}$ and is computed in the following in a system where users of a cell are considered to be uniformly distributed over carriers reflecting a load balancing strategy (i.e. the same load is kept in all carriers). Therefore, the value of the factors $S_{\mathcal{M}_{f}(l), \mathcal{M}_{f}(j)}^{(f)}$ could be written as:

$$
S_{\mathcal{M}_{f}(l), \mathcal{M}_{f}(j)}^{(f)}=\frac{S_{l, j}}{F_{l}}
$$

Then, the coupling matrix and the noise power vector are estimated using (4) and (9):

$$
C_{\mathcal{M}_{f}(j), \mathcal{M}_{f}(l)}^{(f)}=\frac{\frac{S_{l, j}}{F_{l}}}{1-\frac{S_{j, j}}{F_{j}}} \quad P_{N, \mathcal{M}_{f}(j)}^{(f)}=\frac{N_{T}}{1-\frac{S_{j, j}}{F_{j}}}
$$

\section{INTRA-RAT ADVANCED SPECTRUM MANAGEMENT}

\section{A. Problem Formulation}

Spectrum allocations should satisfy QoS requirements that could be reflected by user satisfaction metrics. In general, a user is considered satisfied if its measured $E_{\mathrm{b}} / N_{\mathrm{o}}$ is higher than a given threshold, which in turn is related to certain bit error rate or delay requirements [18]. In this context, outage probability is defined as the fraction of users not achieving the desired $E_{b} / N_{0}$ threshold. In this contribution, we aims at minimizing the outage probability at cell level and more specifically the maximum outage probability over all cells reflecting the intention of operators to minimize the outage probability in each cell and not only in average. This can 
prevent the presence of islands of cells with high probability while the total outage probability is acceptable. We emphasize here that the proposed methodology can be easily adapted to other operator's metrics such as average outage probability or the outage probability of specific services.

Since our objective is to minimize the number of allocated carriers to cells while conserving an acceptable QoS level, we use the following definition of spectrum efficiency when only one service is provided by the operator and that represents cell throughput per unit of bandwidth:

$$
v=R_{b} \frac{1}{K W} \sum_{j \in \Lambda} \frac{\left(1-\mathrm{Pr}_{\mathrm{o}, j}\right) n_{j}}{F_{j}} .
$$

where $W$ is the $5 \mathrm{MHz}$ WCDMA bandwidth, $R_{b}$ is the bit rate of the provided service and $\operatorname{Pr}_{\mathrm{o}, j}$ is the estimation of the outage probably in cell $j$.

With $F_{\text {all }}$ carriers allocated to the operator, the carrier allocation problem aims at finding the best carrier allocation to cells (i.e. sets $\Lambda_{f}$ ) that maximizes the spectrum efficiency and it is expressed by:

$$
\begin{array}{lll}
\text { Maximize } & v \\
\text { Subject to } & \max _{j \in \Lambda} \operatorname{Pr}_{\mathrm{o}, j}<\delta & \\
& F_{j} \leq F_{\text {all }} & \forall j \in \Lambda \\
& P_{T, i_{j}} \leq P_{\max } & \forall i_{j} \in \vartheta_{j}, j \in \Lambda
\end{array}
$$

where $\operatorname{Pr}_{\mathrm{o}, j}$ is the outage probability in cell $j, \delta$ is the outage probability threshold at cell level, $P_{\max }$ is the maximum available power at terminal $i_{j}$ and $P_{T, i_{j}}$ is the required transmitted power by terminal $i_{j}$ and given by:

$$
P_{T, i_{j}}=P_{j, i_{j}} L_{i_{j}, j}
$$

\section{B. ASM Methodology Steps}

Since the outage probability is a non-linear function, the carrier allocation problem defined in (12) is a combinatory problem with non-linear constraints, which is known to be an NP-hard problem [19]. Therefore, we introduce an ASM methodology using simulated annealing principles to avoid local optima. The proposed methodology tests if the allocated number of carriers for the system is sufficient and finds the required number of carriers for each cell.

The algorithm, depicted in the block diagram of Fig. 1, starts in step 1 by estimating global coupling matrix $\boldsymbol{C}$ and noise power $\boldsymbol{P}_{N}$ by using (4) and (5) based on measurements and/or outputs from a planning tool. Step 2 involves the first estimation of the number of carriers to be allocated to each cell. In cell $j$, the needed number of carriers should be sufficient to handle at least the load coming from intra-cell users, which corresponds to parameter $S_{\mathcal{M}_{f}(j), \mathcal{M}_{f}(j)}^{(f)}$. This means that the following condition should be fulfilled [20]:

$$
S_{\mathcal{M}_{f}(j), \mathcal{M}_{f}(j)}^{(f)} \leq 1 \quad \forall f \in \Phi_{j}
$$

where $\Phi_{j}$ is the set of carriers allocated to cell $j$ : $\Phi_{j}=\left\{f: j \in \Lambda_{f}\right\}$.

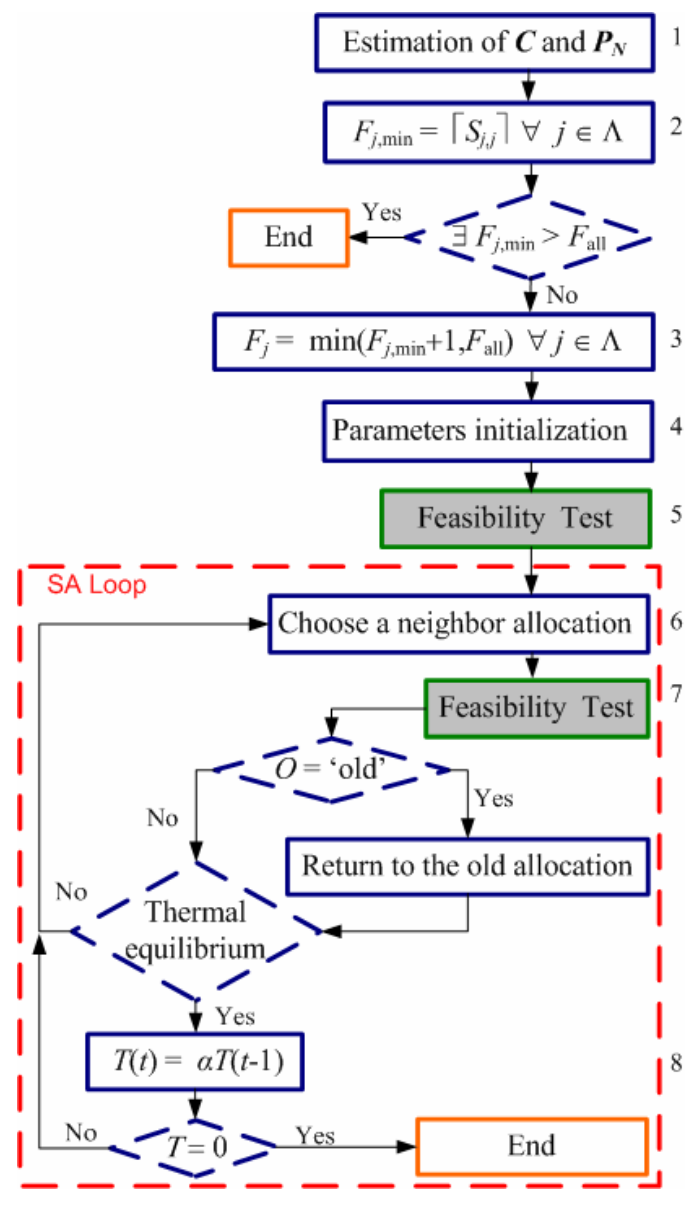

Fig. 1. The block diagram of the proposed ASM methodology

By combining (9) and (14), the estimation of the minimum number of carriers $F_{j \text {,min }}$ for cell $j$ is first initiated using the following equation:

$$
F_{j, \min }=\left\lceil S_{j, j}\right\rceil \text {. }
$$

where $\left\lceil S_{j, j}\right\rceil$ is the first integer higher or equal than $S_{j, j}$.

Then, we test if the system cannot handle just the intra-cell interference using $F_{\text {all }}$ (i.e. there exists at least one cell that require a number of carriers higher than the available number). In this case, the algorithm will stop without saving any solution in the database, meaning that the network would require more carriers for a proper operation. Otherwise, we add one carrier to each cell in step 3 as a margin for inter-cell interference if the allocated number of carriers to the cell is lower than $F_{\text {all }}$. Hence, the initial value of $F_{j}$ is given by:

$$
F_{j}=\min \left(\left\lceil S_{j, j}\right\rceil+1, F_{\text {all }}\right)
$$

In step 4, we start with an allocation where the first carrier will be allocated to all cells, the second to all cells requesting at least two carriers, the third to all cells requesting at least three carriers, and so on. The solution is tested, in step 5, using the feasibility test function that is explained in section III-C in order to see if the allocation is within the solution space and save it in the database of the algorithm as best 
solution. Feasibility test output includes the new outage probabilities in all cells, the spectrum efficiency and string $O$ that is assigned to 'new' if the allocation at iteration $t$ should be conserved or 'old' if the allocation at iteration $t-1$ should be used since the allocation of the actual iteration does not increase the spectrum efficiency or does not satisfy QoS constraints.

Then, the algorithm enters in the simulated annealing loop where the solution space involves all carrier-to-cell allocations that verify the following two constraints:

$$
\begin{array}{ll}
F_{j} \leq F_{\text {all }} & \forall j \in \Lambda \\
F_{j} \geq F_{j, \min } & \forall j \in \Lambda
\end{array}
$$

It is worth here to define the different parameters of the simulated annealing meta-heuristic [16]. The neighborhood structure $\mathcal{N}_{\mathrm{e}}(t)$ at iteration $t$ is built by adding/removing one carrier of the allocated carriers to a cell in the solution space. The cost function of an allocation at iteration $t$ is defined by $c(t)=-v(t)$. The transition probability is the probability of accepting the allocation at iteration $t$ by comparing its cost function with the cost function of the previous allocation and it is defined by:

$$
\operatorname{Pr}(t, t-1)=\min \left[1, \exp \left(\frac{c(t-1)-c(t)}{T(t)}\right)\right]
$$

where $T(t)$ is the temperature at iteration $t$. This temperature is decreased using a cooling function each time the thermal equilibrium is reached and when it reaches 0 , the algorithm stops. The cooling function is given by $T(t)=\alpha T(t-1)$, where $\alpha$ is a predefined constant $(0<\alpha<1)$. The thermal equilibrium is reached after a number of iterations that depends on the temperature. It is set at the beginning to be equal to the number of cells in order to allow all cells to change their configuration. Then, this number is reduced each time the thermal equilibrium is reached by a factor of $T(t)$.

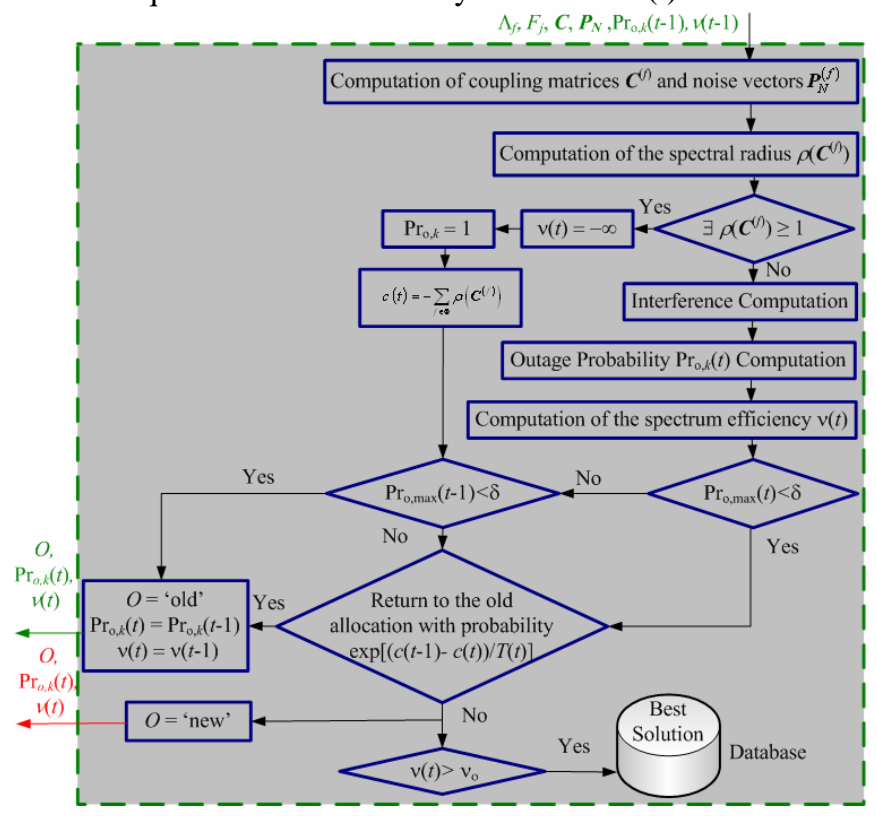

Fig. 2. Feasibility test diagram
The transition probability always allows changing the allocation if the cost function is decreased. Moreover, it allows this change in case of an increasing cost function with a probability less than unity to prevent the convergence to local optima.

At step 6, the algorithm chooses randomly an allocation from $\mathcal{N}_{\mathrm{e}}(t)$. The feasibility test is applied to the new allocation at step 7 and the new allocation is conserved/discarded upon the output of the test.

At step 8, the temperature is decreased if the thermal equilibrium is reached. Then, a new iteration is executed if the temperature did not reach 0 . Otherwise, the algorithm will end with an output extracted from the database and including the number $F_{j}$ of carriers allocated to cell $j$ and the sets $\Lambda_{f}$.

\section{Feasibility Test}

In order to satisfy QoS requirements, the operator should specify a feasibility test together with the required thresholds. The feasibility test is shown in Fig. 2 and is based on estimating the maximum outage probability and maximizing the spectrum efficiency. The inputs of the feasibility test are the sets of cells $\Lambda_{f}$ sharing the same carrier, the number of carriers allocated to each cell $F_{j}$, coupling matrix $\boldsymbol{C}$, noise power vector $\boldsymbol{P}_{N}$, the spectrum efficiency at the previous iteration $v(t-1)$ and the outage probabilities at the previous iteration $\operatorname{Pr}_{0, k}(t-1)$.

At the initialization, the algorithm computes carrier's coupling matrices and noise power vectors using (10) for all carriers. Then, the spectral radius $\rho\left(C^{(f)}\right)$ of each carrier's coupling matrix is computed.

If one spectral radius is higher than 1 , the system is not feasible according to the conclusions of [13], meaning that it is not possible to provide the required services to the users in the different cells. In this case, we set the spectrum efficiency to $-\infty$, the outage probability of all cells to unity and the cost function to $c(t)=-\sum_{f \in \Phi} \rho\left(\boldsymbol{C}^{(f)}\right)$. Then, if the maximum outage probability over cells $\operatorname{Pr}_{\mathrm{o} \text {,max }}(t-1)$ was lower than $\delta$ in the previous iteration, the new allocation is discarded. In this case, the feasibility test output $O$ is set to 'old'. Otherwise, the new allocation is conserved depending on the transition probability and output $O$ is set to 'new'.

On the contrary, if all spectral radius are lower than 1 , interference vectors $\boldsymbol{I}^{(f)}$ are estimated using (3) by replacing $\boldsymbol{C}$ and $\boldsymbol{P}_{N}$ by $\boldsymbol{C}^{(f)}$ and $\boldsymbol{P}_{N}^{(f)}$ :

$$
\boldsymbol{I}^{(f)}=\left(\mathfrak{I}_{\left|\Lambda_{f}\right|}-\boldsymbol{C}^{(f)}\right)^{-1} \boldsymbol{P}_{N}^{(f)}
$$

where $\mathfrak{I}_{\left|\Lambda_{f}\right|}$ is the $\left|\Lambda_{f}\right| \times\left|\Lambda_{f}\right|$ identity matrix.

Then, the outage probability $\operatorname{Pr}_{\mathrm{o}, k}^{(f)}(t)$ in carrier $f$ and the total outage probability $\operatorname{Pr}_{\mathrm{o}, k}(t)$ of each cell $k$ are estimated using (22) and (23) of the appendix. The estimated outage probabilities are then used to compute the spectrum efficiency $v(t)$. Maximum outage probability $\operatorname{Pr}_{\mathrm{o}, \max }(t)=\max _{k \in \Lambda}\left(\operatorname{Pr}_{\mathrm{o}, k}(t)\right)$ is compared to threshold $\delta$. If $\operatorname{Pr}_{0, \max }(t)$ is higher than $\delta$ and 
$\operatorname{Pr}_{0, \max }(t-1)$ is lower than the threshold, the new allocation should be discarded and the feasibility test output $O$ is set to 'old'. If $\operatorname{Pr}_{0, \max }(t)$ and $\operatorname{Pr}_{0, \max }(t-1)$ are both higher than threshold $\delta$, the new allocation is conserved with a probability $\operatorname{Pr}(t, t-1)$. The new allocation is conserved also in the same manner if $\operatorname{Pr}_{0, \text { max }}(t)$ is lower than threshold $\delta$. In the last case, if the spectrum efficiency $v(t)$ is higher than the saved best value, the database is updated by the new allocation parameters as best solution over all previous iterations. In all these cases, the feasibility test sets output $O$ to 'new' and returns the decided allocation together with the corresponding $\mathrm{Pr}_{\mathrm{o}, j}, v$ and $F_{j}$.

\section{PRACTICAL CONSIDERATIONS}

The ASM methodology could be applied either in the planning phase or dynamically executed as a response to relevant changes in the traffic distribution using appropriate triggering events (e.g. based on QoS indicators, etc.). In the first case, the operator identifies different periods of time with different traffic distribution and associates a different frequency allocation based on a specific coupling matrix for each period. These periods are rather medium and long-term periods (e.g. hours, half a day, etc.) and the elements of the matrix are estimated from the expected averaged values. In this context, the fast fluctuation (due to mobility, fast fading, etc.) is averaged and does not have important impact on the accuracy of the matrix. For the same reason the proposed methodology does not put significant computation constraints due to scalability since computations are only required at the rather slow medium/long-term traffic pattern variability. Moreover, all the needed information $\left(E_{\mathrm{b}} / N_{\mathrm{o}}\right.$, long-term path losses, etc.) in the dynamic version for the computation of the coupling matrices can be obtained using the measurements collected either by cells or mobiles in an operative network.

\section{Simulations AND RESUlts}

The simulation layout and simulation parameters are introduced in Fig. 3 and Table 1. We assume that the operator has three carriers at the beginning of simulations $\left(F_{\text {all }}=3\right)$. The used value of $\alpha$ and $T(0)$ are chosen to be the values that leads to the fast convergence based on simulation results that are not shown here.

Table 1. Simulation Parameters

\begin{tabular}{|l|l|}
\hline Cell radius & $1 \mathrm{~km}$ \\
\hline Path loss model & $128.1+37.6 \times \log _{10} d(\mathrm{Km})$ \\
\hline Background noise power & $-103 \mathrm{dBm}$ \\
\hline Maximum allowed power & $21 \mathrm{dBm}$ \\
\hline Transmitted power range & $61 \mathrm{~dB}$ \\
\hline$E_{\mathrm{b}} / N_{\mathrm{o}}$ target & $3 \mathrm{~dB}$ \\
\hline Spreading factor $\Theta$ & $23 \mathrm{~dB}$ \\
\hline Shadowing factor deviation & $7 \mathrm{~dB}$ \\
\hline Shadowing factor cross-correlation & 0.5 \\
\hline Power control & Perfect power control \\
\hline$T(0)$ & 0.5 \\
\hline$\alpha$ & 0.8 \\
\hline
\end{tabular}

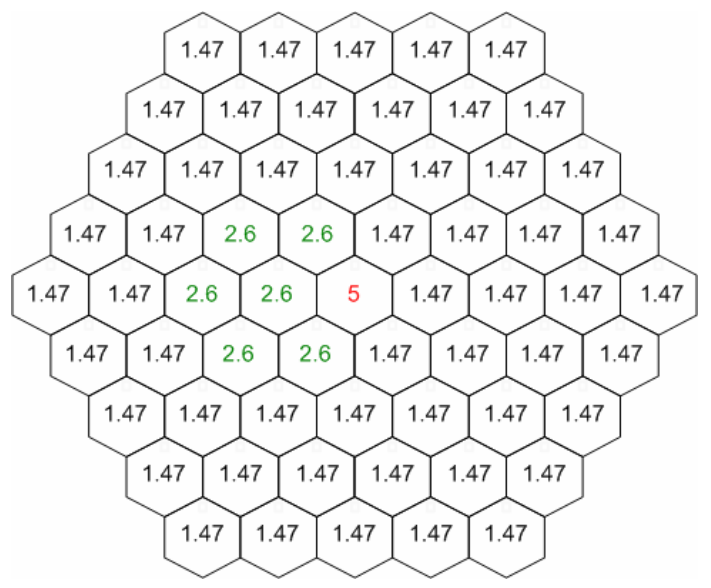

Fig. 3. Simulation layout (numbers inside cells are the percentage of users in each cell in respect to the total number of users)

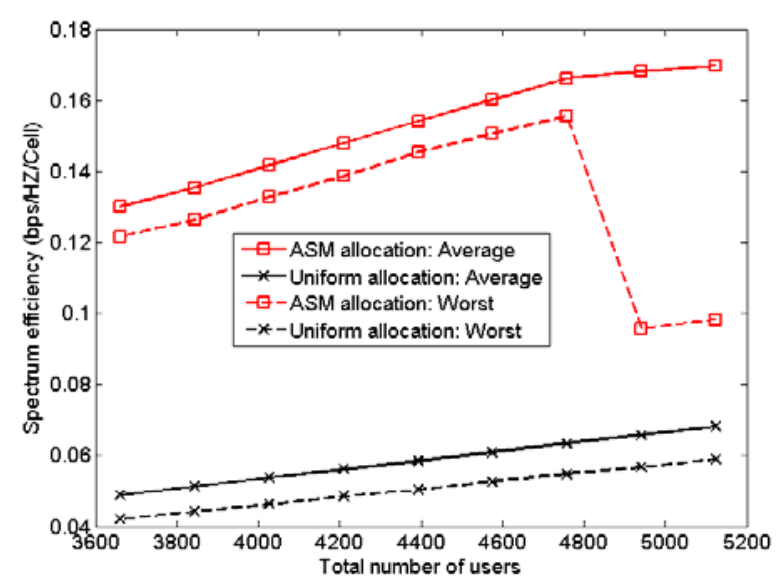

Fig. 4. Average and worse spectrum efficiency

The results of the proposed ASM methodology are compared to the uniform carrier allocation methodology that consists of uniformly distributing the three carriers over cells whatever the cell loads are (i.e. always $F_{j}=F_{\text {all }}$ ). Simulation results showed that the proposed allocation, as well as the proposed uniform allocation, is able to keep the maximum outage probability lower than $\delta$ when it is possible (i.e. the number of carriers are sufficient to handle the requested traffic) and to detect system capacity limit, which is reached when the maximum outage probability is higher than $\delta$. These results are not shown here for brevity.

The average spectrum efficiency $v$ over all cells is plotted in Fig. 4 as a function of the total number of mobiles. The considered service is a circuit-switched service with $R_{b}=12.2$ Kbps useful data rate. The ASM methodology allows very high spectrum efficiency compared to the efficiency of the uniform allocation (i.e. more than the double) and keeps the variation of cell's spectrum efficiency as low as the discreet values of the bandwidth allows. This is due to the fact that it is able to find the minimum number of carriers needed by a cell while keeping QoS levels of users at the required level. Moreover, we show in Fig. 4, the minimum spectrum efficiency over all cells (i.e. worst case) which appears in cells 
with low loads. We can notice also in this case the high difference between the two methodologies due to the fact that the uniform allocation always associates three carriers to each cell even if the cell load is very low. The maximum spectrum efficiency over all cells, which is not plotted for simplicity, is the same for both methodologies since it appears in the central cells where three carriers are allocated due to high traffic load.

In Fig. 5, we show the percentage of cells using 1, 2 and 3 carriers respectively when the ASM methodology is used. As we can see, the majority of cells uses one carrier and the number of these cells as well as the number of cells using three carriers is constant until the system reaches its capacity limits. In Fig. 6, we show an example of carrier distribution over cells as well as the number of free carriers in each cell represented by grayscale map (i.e. white: 2 free carriers, gray: 1 free carrier, black: no free carriers). It is interesting to emphasize here the capability of the ASM methodology of liberating carriers in some cells. These carriers could be used by another RAT/operator or opportunistically by cognitive radios.

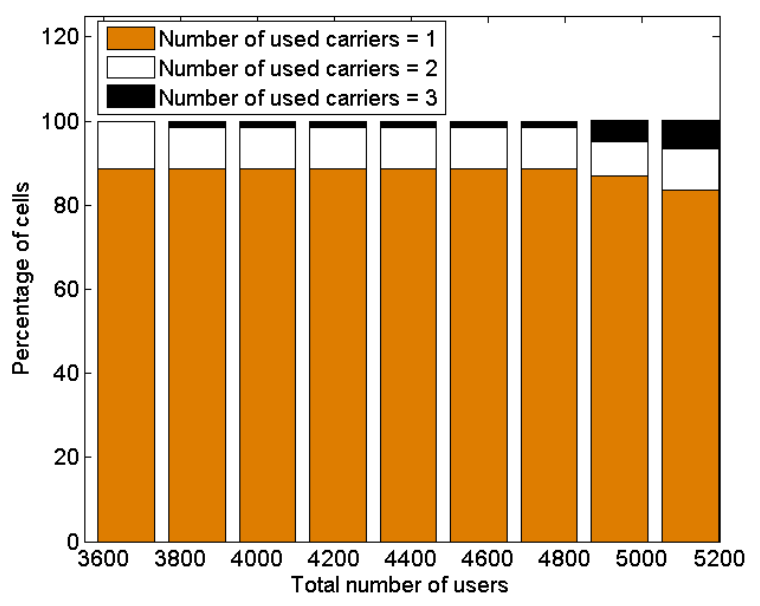

Fig. 5. Distribution of used number of carriers by cells

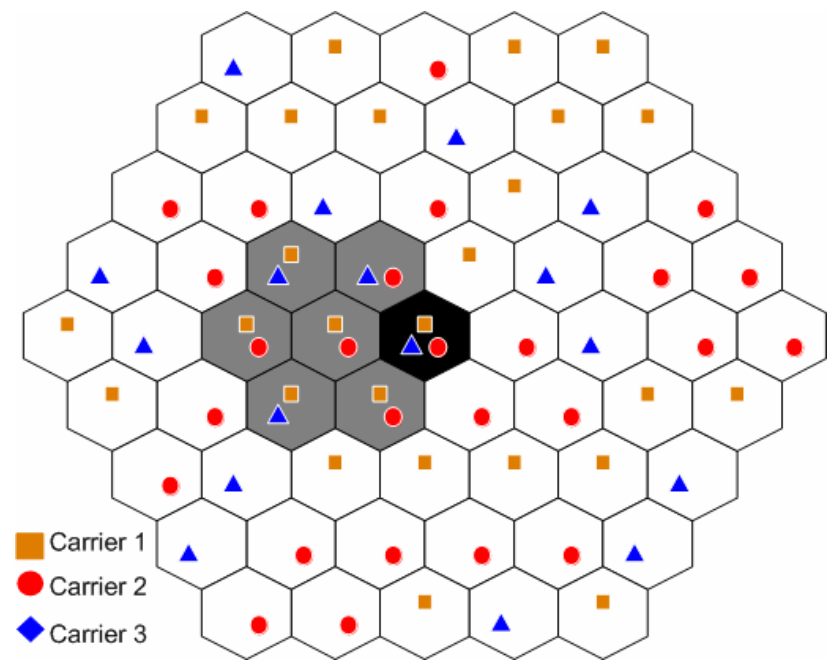

Fig. 6. Carrier distribution and the number of free carriers in each cell for a system of 3843 mobiles

\section{CONCLUSIONS}

In this paper, we have introduced a centralized ASM methodology for WCDMA networks using simulated annealing. The proposed approach is based on the utilization of coupling matrix properties to reduce inter-cell interactions. The main objective of the proposed methodology is to minimize the number of needed carriers in a cell-by-cell approach satisfying mobile's QoS levels. This approach leads to the release of several carriers in some geographical areas where opportunistic access to the spectrum can be allowed to cognitive radios. Simulation results have shown that the proposed methodology leads to interesting results in terms of guaranteeing QoS levels reflected by outage probability and increasing spectrum efficiency.

Our future work focuses on the utilization of the released carriers by cognitive radios without polluting WCDMA users with harmful interference and the extension of the ASM methodology to take into account different RATs such as OFDMA-based RATs.

\section{APPENDIX: Estimation OF THE OUTAGE PROBABILITY}

Outage probability estimation could be used by the ASM algorithm to find the best spectrum allocation using the minimum number of carriers. The definition of this indicator is relevant for ASM since it estimates the real performance of the spectrum allocation algorithms without testing them on real systems, which could lead to unacceptable performance for significant periods of time. The proposed estimation uses the coupling matrix to compute vector $\boldsymbol{I}$.

We assume that a mobile $i_{j}$ is in outage for a given vector of total received power $\boldsymbol{I}$ if the necessary transmitted power $P_{T, i_{j}}$ for achieving the target $E_{\mathrm{b}} / N_{\mathrm{o}}$ is higher than the maximum acceptable power. Therefore, the estimated outage probability $\operatorname{Pr}_{\mathrm{o}, j}$ in cell $j$ could be given by:

$$
\operatorname{Pr}_{\mathrm{o}, j}=\operatorname{Pr}\left(P_{T, i_{j}}>P_{\max } \mid i_{j} \in \vartheta_{j}\right)
$$

where $\vartheta_{j}$ is the set of users connected to cell $j$.

Having $\boldsymbol{I}$, outage probability could be estimated using path loss distribution of mobiles towards their servers. From (1), (2) and (13), we can write

$$
P_{T, i_{j}}=I_{j} L_{i_{j}, j} / \varepsilon_{i_{j}}
$$

If we assume that all users require the same service (i.e $\varepsilon_{i_{j}}$ is the same for all users and set to $\varepsilon$ ), then the outage probability in (20) can be written using (21) as

$$
\begin{aligned}
\operatorname{Pr}_{\mathrm{o}, j} & =\operatorname{Pr}\left(L_{i_{j}, j}>\frac{\varepsilon}{I_{j}} P_{\max } \mid i_{j} \in \vartheta_{j}\right) \\
& =1-\operatorname{cdf}_{j}\left(\varepsilon P_{\max } / I_{j}\right)
\end{aligned}
$$

where $\mathrm{cdf}_{j}$ is the cumulative distribution function of mobile path losses served by cell $j$. In scenarios where $j$ is associated to several carriers, the outage probability of cell $j$ is given by:

$$
\operatorname{Pr}_{\mathrm{o}, j}=\frac{\sum_{f \in \Phi_{j}} \operatorname{Pr}_{\mathrm{o}, j}^{(f)} n_{j}^{(f)}}{n_{j}}
$$


where $n_{j}^{(f)}$ is the number of users in cell $j$ associated to carrier $f$ and $\operatorname{Pr}_{\mathrm{o}, j}^{(f)}$ is the outage probability in cell $j$ within carrier $f$ computed using (22).

The cumulative distribution function of mobile path losses toward the serving cell could be analytically estimated as in [21], or can be statistically collected from the system measurements of path losses. The latter is better due to the fact that different types of traffic distribution will have different types of path loss distributions that can be very difficult to tackle with the analytical model. Therefore, we use in this paper the statistical method for path loss distribution.

In the following, we highlight the tight relation between the estimated and the measured outage probabilities using system level simulations. In Fig. 7, the estimated outage probabilities of all cells for one carrier are plotted together with the measured outage probabilities in two scenarios: heterogeneous scenario with the cell load distribution of Fig. 3 and homogeneous scenario where all cells have the same load. The complete radio parameters can be found in Table 1. We can see that the estimated outage probability gives a good approximation of the measured values in both homogeneous and heterogeneous traffic distribution over cells.

\section{ACKNOWLEDGMENTS}

This work was performed in project E2R2/E3 which has received research funding from the Community's Sixth/Seven Framework program. This paper reflects only the authors' views and the Community is not liable for any use that may be made of the information contained therein. The contributions of colleagues from E2R2/E3 consortium are hereby acknowledged. This work has also been supported by the Spanish Research Council under COGNOS grant (ref. TEC2007-60985)

\section{REFERENCES}

[1] M. M. Buddhikot, P. Kolodzy, S. Miller, K. Ryan, and J. Evans, "DIMSUMNet: New Directions in Wireless Networking Using Coordinated Dynamic Spectrum Access," IEEE WoWMoM, 2005

[2] A. K. Maitra, Wireless Spectrum Management: Policies, Practices and Conditioning Factors, Mc Graw-Hill, June 2004

[3] J.M. Peha, "Approaches to spectrum sharing," IEEE Communications Magazine, vol.43, $\mathrm{n}^{\circ}$ 2, 2005, pp. 10- 12

[4] E2R Deliverable D5.3, Algorithms and Performance, including FSM \& RRM/Network Planning, July 2005

[5] I. F. Akyildiz, W. Lee, M. C. Vuran, and S. Mohanty, "NeXt generation/dynamic spectrum access/cognitive radio wireless networks: a survey," Computer Networks: The International Journal of Computer and Telecommunications Networking, vol 50, 2006, p.p. 2127 - 2159

[6] D. Grandblaise, K. Moessner, P. Leaves and D. Bourse, "Reconfigurability support for dynamic spectrum allocation: from the DSA concept to implementation," IEEE SympoTIC03, 2003, pp. 9 - 12
[7] M. Buddhikot and K. Ryan, "Spectrum management in coordinated dynamic spectrum access based cellular networks" IEEE DySpan, 2005

[8] L. Kovacs \& A. Vidacs, "Spatio-Temporal Spectrum Management Model for Dynamic Spectrum Access Networks," TAPAS 2006

[9] FCC, "Spectrum Policy Task Force seeks public comments on issues related to commission's spectrum policies," ET Docket No. 02-135, 2002

[10] T. A. Weiss and F. K. Jondral, "Spectrum pooling: an innovative strategy for the enhancement of spectrum efficiency," IEEE Communications Magazine, 2004, pp. 8- 14

[11] M. K. Pereirasamy, J. Luo, M. Dillinger and C. Hartmann "Dynamic Inter-Operator Spectrum Sharing for UMTS FDD with Displaced Cellular Networks," IEEE WCNC05, 2005, 3, p.p.1720- 1725

[12] M. Bennis and J. Lilleberg, "Inter-Operator Resource Sharing for 3G Systems and Beyond," IEEE ISSTA, 2006, p.p. 401-405

[13] J. Nasreddine, O. Sallent, J. Pérez-Romero, R. Agusti, "Novel Inter-Cell Interaction Approach for WCDMA-based Cognitive Networks", IEEE ICC, Glasgow-Scotland, June 2007

[14] J. Nasreddine, J. Pérez-Romero, O. Sallent, R. Agusti ,"Dynamic Spectrum Management Methodology for WCDMA Systems Based on InterCell Interaction Approach", WPMC, September 2006

[15] — , "Intra-RAT Spectrum Management Methodology for WCDMA Systems Encompassing Uplink and Downlink," IEEE WCNC, 2007.

[16] S. Kirkpatrick, C. D. J. Gellat, and M. P. Vecchi, "Optimization by simulated annealing,” Science, vol. 220, pp. 671-680, 1983

[17] S. A. Grandhi, R. Vijayan, D. J. Goodman, and J. Zander, "Centralized Power Control in Cellular Radio Systems," IEEE Transactions on Vehicular Technology, vol. 42, pp.66 - 468, 1993

[18] J. B. Kim and M. L. Honig "Resource Allocation for Multiple Classes of DS-CDMA Traffic," IEEE Trans. on Veh. Tec., 2000, p.p. 506 - 519

[19] M. R. Garey and D. S. Johnson, Computers and Intractability: A Guide to the Theory of NP-Completeness. W. H. Freeman and Co., 1979

[20] J. Pérez-Romero, O. Sallent, R. Agusti and M. A. Diaz-Guerra, Radio Resource Management strategies in UMTS, John wiley \& Sons, 2005

[21] J. Pérez-Romero, O. Sallent, R. Agusti, "Impact of Indoor traffic on WCDMA capacity," PIMRC, 2004, vol. 4, pp. 2861- 2865

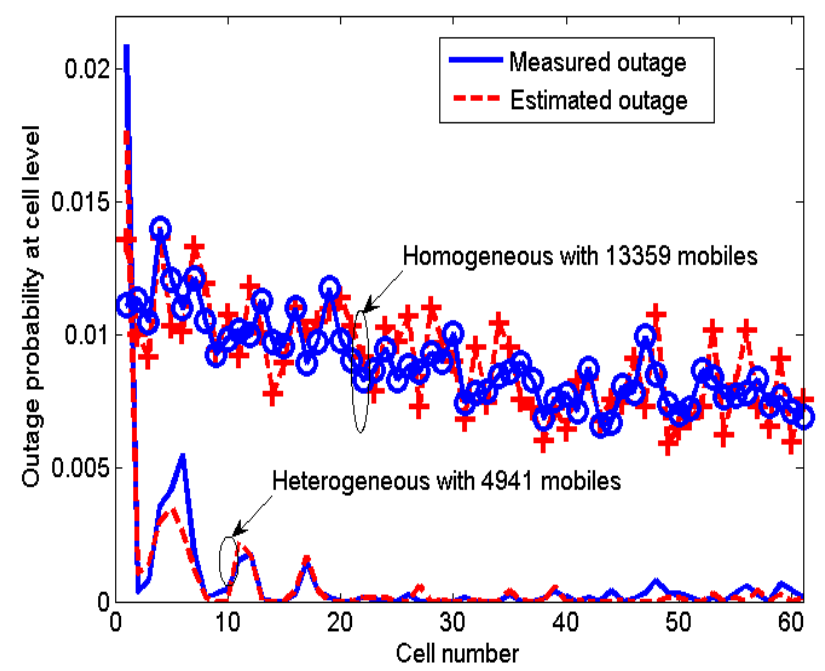

Fig. 7. The outage probabilities of all cells for two traffic densities 